\title{
Preservice Teachers' Applications of Integrated Design Models for Teaching and Learning of Solids
}

\author{
Clement Ayarebilla Ali \\ University Of Education, Winneba, Post Office Box 25, Winneba-Ghana
}

Received: May 03, 2016 / Accepted: June 01, 2016 / Published: October 25, 2016.

\begin{abstract}
This study integrated instrumental and relational approaches to the teaching and learning of solids in a preservice teacher's class. The researcher guided the preservice teachers to gather various prisms, pyramids, and spheres to study the total surface areas and volumes. At the end of the integrated models, the test scores showed closed relationships in the concurrent instructional strategies of the integrated models. The researcher therefore, recommended the design models for the teaching and learning of solids in mathematics.
\end{abstract}

Keywords: integrated design model; preservice teachers; relational and instrumental; three-dimensional figures

\section{Introduction}

The study of three-dimensional figures (mensuration) is the science of measuring solid figures or solids. The solids are mainly categorized into regular and irregular, prisms and pyramids, and spheres. Irrespective of these categorizations, the common concepts of interest to preservice teachers are total surface areas and volumes, and the procedures of discovering these two concepts are collecting, classifying, testing, and making inferences. Quite apart from these procedures, it is not still clear the appropriate mathematical design and the associated type of learning expected of preservice teachers. This seeming uncertainty breeds mathematical anxiety, weak knowledge and computational errors in applying solids to solve problems of the society. This opens up the debate as to whether instrumental or relational or both types of learning should be espoused by preservice teachers, and extends the contention to the various philosophical viewpoints. This paper therefore, seeks to explore the philosophical viewpoints and situate an integrated design model for teaching and

Corresponding author: Clement Ayarebilla Ali, University Of Education, Winneba, Post Office Box 25, Winneba-Ghana. learning of solids (Bishop, 1980; Bolt, 1987; Asiedu, 1997; Asafo-Adjei, 2002; Appau, 2006).

\section{Postpositivist Views of Mathematical Knowledge}

This view is sometimes called the absolutism, logicism, scientific method, empirical science, and postpositivism. The absolutist view of mathematical knowledge assumes certainty and unchallengeable truths. It is called post-positivism because it represents the thinking after positivism, challenging the traditional notion of the absolute truth of knowledge and recognizing that everyone cannot be positive about knowledge when studying the behaviour and actions of humans. It is logicism because it represents all the concepts of mathematics that can be ultimately reduced to logical sets of interrelated theories, mathematical truths, axioms and rules (Ernest, 1991; Summit \& Rickards, 2013; Creswell, 2014).

Applying this view to solids, one can state, for instance, that the total surface area of a cube is $6 x^{3}$ because a cube is a cuboid whose sides are equal. It is also verifiable that the volume of a cone is one-third the volume of a cylinder. In both of these instances, one can observed elements of instrumental and 
relational learning. Thus, knowing the total surface area of cuboid is instrumental and inking it to a cube is relational (Skemp, 1976).

\section{Constructivist Views of Mathematical Knowledge}

The constructivists view mathematical knowledge in two-fold claims of intuitive positivity i.e. (construing mathematical notions and logical operations is coherent and legitimate) and negativity (classical way of construing mathematical notions and logical operations is incoherent, illegitimate, distorted, and unintelligible. Constructivism constantly seeks understanding, develops subjective learning experiences, and makes sense of socio-historical perspectives (Ernest, 1991; Crotty, 1998; South African Institute for Distance Education--SAIDE, 2008; Summit \& Rickards, 2013).

The two main schools of constructivism are radical and social. Radical constructivists totally discard the notion of truth and propose viability to make accurate predictions, to solve problems, and to accomplish personal goals. Social constructivism, on the other hand, focuses on knowledge sharing among members of the classroom community in an interactive fashion. Social constructivism claims radical constructivism does not take into account the role of social interaction in the construction of knowledge (Beswick, 2005; Egodawatte, 2011).

It can be deduced here in this view, that solving for the total surface area (A) of pyramid, for instance is both radical and social. Radical in the sense one needs to perform activities to discover that $A=\frac{n}{2} b h+$ area of base face, where $\mathrm{n}$ is the number of sides of the base face, and it is social in the sense that there should be adequate socio-cultural knowledge of pyramids to promote the discovery. It should be noted that instrumental knowledge of area of a triangle, the basic polygon is crucial to relate the triangle to other pyramids (SAIDE, 2008).

\section{Pragmatic Views of Mathematical Knowledge}

Pragmatism arises out of actions, situations, and consequences rather than antecedent conditions rigidly enshrined in postpositivism and constructivism. Instead of focusing on methods, the pragmatists emphasize on the problem and implore every available strategy to unravel the problem and advance its solution. The pragmatist is not committed to any one system of philosophy, individuals have the freedom of choice, and theoretical lenses are open to multiple scenarios to tackle a problem (Creswell, 2014).

Therefore, most mathematical learning should assume the mixture of the two ways of learning especially when both are appropriate and useful. For instance, it is practically impossible to prove that the total surface area of a sphere is $\mathrm{A}=4 \pi r^{2}$ if there is no activity to derive it from the area of a circle. And of course, getting the area of the circle is instrumental whereas extending that knowledge to derive the total surface area of the sphere is relational. Stakeholders therefore, need to explore various ways of instituting integrated models for concepts in mathematics (Skemp, 1978).

\subsection{Integrated Design Model for Relational and Instrumental Learning}

Concrete materials used to model mathematical concepts are important tools for helping children to learn and to connect the model to the concept represented. And one cannot foster model building without having the relationship in mind. Models are the pictorial representations of physical concepts, and integrated models bring different sets of physical phenomena into one meaningful whole to ease a common understanding. It is therefore prudent to commence model building processes from simple to complex relations without loss of generality (SAIDE, 2008).

Instrumental learning refers to the ability to formulate, organize, and apply algorithms without 
assigning reasons to the process. The merits of instrumental learning are that it is easier to understand, the rewards are more immediate, and one often gets the right answer more quickly. Relational understanding, on the other hand, refers to the ability to gather materials, abstract ideas, reorganize concepts and contextualize the knowledge to given situation. Relational mathematics, on the other hand, is more adaptable to new tasks, easier to remember, a goal in itself, and organic in quality (Skemp, 1976; Skemp, 1978; Summit \& Rickards, 2013).

Pesek and Kirshner (2000) bemoan the cognitive interference (previous understandings), and the attitudinal interference (previously acquired opinions and attitudes) block the process of engaging learners in undertaking instructional activities. That is why the integrated designed model is necessary to cater for relational challenges in long time of engagement, too difficult to understand, complex transfer of knowledge, and heavily dependence on schemas of the learner. In most learning situations, relational learning could be affected by effects of examinations, over-burdened syllabi, difficulty of assessments, and psychological difficulty for teachers to re-structuring their existing schemas. On the other hand, instrumental could be hindered by lack of exploration, inadequate investigative discovery, and no interaction of learners to maximize learners' potentials. It is therefore, clear that the two types of learning concurrently interplay in the classroom setting (Skemp, 1976; Pesek \& Kirshner, 2000; SAIDE, 2008).

\subsection{Integrated Design Model for Three-Dimensional Figures}

In employing the integrated design model, the solids are categorized into polyhedra (flat-surfaced polygons comprising platonic, prisms and pyramids) and non-polyhedra (mix-surfaced figures comprising sphere, cylinder and cone). In particular, prism is a solid figure whose two bases are parallel and congruent polygons, and named according to the shape of the base, such as triangular, rectangular, pentagonal, and hexagonal prisms to represent three, four, five and six-sided prisms respectively. The cube is a special rectangular prism whose faces are squares, and the cylinder is a special prism whose faces connected by parallel curved surfaces.

A pyramid is a polyhedron whose base is a polygon and whose other faces are triangles that meet at a common vertex, and are equally named by the shape of the base, vis-à-vis triangular (tetrahedron), rectangular, hexagonal pyramids, and octagonal pyramids. The cone is a special pyramid whose base is circular base. A sphere is a three dimensional non-polyhedron, which is made up of all points in the space, which lie at a constant distance (radius, or r), from a fixed point called the centre of the sphere (Asiedu, 1997; Asafo-Adjei, 2002; Appau, 2006; Ontario Ministry of Education, 2008; The Improving Mathematics Education in Schools-TIMES, 2011).

Any integrated design model should concurrently encompass these relations in systematic and sequential order to meet the two main groups of learners. In polyhedra for instance, the simplest prism is the tetrahedron of four triangles, and the simplest pyramid is the triangular prism of four triangles. The irregular figures provide links between hemispheres and spheres, spheres and circles, and cylinders and spheres (Asafo- Adjei, 2002; Appau, 2006; Ontario, 2008).

\subsection{Methods}

The main objective in this study was to devise an integrated design models for concurrent instructions in the mathematics classroom. The researcher simultaneously used both models on 122 preservice teachers, where the instrumental models served as the control. The interval between the instrumental and relational was so close to guarantee internal validity for the effectiveness of the integrated design models (Creswell, 2014).

In using integrated models, the researcher guided the participants to first group the solids into prisms, 
pyramids and spheres, after which they were again guided to recall, memorize and apply routine algorithms to solve problems in total areas and volumes. After the three hours, they were guided to draw shapes of the solids, and derive appropriate relations between any two sets of solids to solve concurrent problems in the same total surface areas and volumes. The researcher, and conjunction with the preservice teachers, constructed three separate tables, to synchronize and analyze these relations among any pairs of prisms, pyramids and spheres so as to compare the knowledge achieved in instrumental and the relational learning. The results of the pairs were outlined and discussed below.

\subsection{Results}

The results of table 1 show the order in which preservice teachers grouped the prisms in the integrated design models. They applied the instrumental knowledge and extended the useful ideas to the relational ones. In the instrumental, participants started with cuboids by gathering the formulas for the total surface areas of a cuboid of length 1 , breadth $b$ and height $h$ as $A=2(l b+b h+h l)$, cube as $A=6 l^{2}$, and cylinder as $A=2 \pi r(r+h)$. The volumes of these respective prisms are $\mathrm{V}=\mathrm{l} \times \mathrm{b} \times \mathrm{h}$, a cube as $\mathrm{V}=1^{3}$, and cylinder as $\mathrm{V}=\pi \mathrm{r}^{2} \mathrm{~h}$.

In the relational ones, preservice teachers' built the total surface area of the cuboid as the sum of area of rectangle $1(1 \times h)+$ area of rectangle $2(1 \times b)+$ area of rectangle $3(1 \times \mathrm{h})+$ area of rectangle $4(\mathrm{l} \times \mathrm{b})+$ area of rectangle $5(\mathrm{~b} \times \mathrm{h})+$ area of rectangle $6(\mathrm{~b} \times \mathrm{h})$, and sum up to $2(1 \times b)+2(b \times h)+2(1 \times h)$ or $2(l b+b h+h l)$. A cuboid, whose length, breadth and height are all equal is called a cube of edge 1 , and the total the surface area as $2(1 \times 1+1 \times 1+1 \times 1)$ or $61^{2}$.

Instrumentally, the lateral surface area of a cuboid $A=2 l h+2 b h$ or $2(1+b) h$ or $4 \times\left(\pi r^{2}\right)$, and that of the cube is $A=41^{2}$. Relationally, the curved surface area of the cylinder is the area of the rectangular sheet (lxb), which is the perimeter of the base of the cylinder $\times \mathrm{h}$ or $2 \pi \mathrm{r} \times \mathrm{h}$. And the area of the two circular bases is $2 \pi \mathrm{r}^{2}$, and this gives the total surface area of the cylinder as $\mathrm{A}=2 \pi r h+2 \pi r^{2}$ or $2 \pi r(h+r)$. The area of a rectangle is denoted by $\mathrm{A}$, the height up to which a number of rectangles are stacked is $h$, and the volume of the cuboid is designated as V. This gives the volume of the cuboid to be $\mathrm{A} \times h$. This means volume of a cuboid=base area $\times$ height or $1 \times b \times h$. The volume of the cube is the number of cubes of edge of unit length 1 and this translates into $\mathrm{V}=$ edge $\times$ edge $\times$ edge $=1^{3}$. And just as a cuboid or cube is built up with rectangles or squares of the same size, the circular cylinder can also be built up using circles of the same size. So, the volume of a cylinder can be obtained as base area $\times$ height $=$ area of circular base $\times$ height $=\pi r^{2} h$.

The results of table 2 show the order in which preservice teachers grouped the pyramids the integrated design model. They started with a tetrahedron, and extended the ideas to the rectangular, and the pentagonal pyramids, and generalize it to any pyramid of number of side $n$. Instrumentally, the total surface area of the tetrahedron recalled was $A=\frac{3}{2} b h+$ base area of triangle, rectangular pyramid was $\quad A=\frac{4}{2} b h+$ base area of rectangle , and pentagonal pyramid was $A=\frac{5}{2} b h+$ base area of pentagon. Relationally, the total surface area of a tetrahedron was $A=\frac{3}{2} b h+\frac{1}{2} b h \quad$ or $\quad 2 b h, \quad$ the rectangular is $A=\frac{4}{2} b h+\frac{2}{2} b h$ or $3 b h$, and the pentagonal was $A=\frac{5}{2} b h+\frac{3}{2}$ or $4 \mathrm{bh}$, and hence any other pyramid of higher dimension was $A=(n-1) b h$.

The volume of the tetrahedron is $V=\frac{1}{3} A h$, rectangular pyramid is $V=\frac{1}{3} h(A 1+A 2)$, pentagonal is $V=\frac{1}{3} h(A 1+A 2+A 3)$, and hence the volume of any other higher dimension was $V=\frac{1}{3} h\left(\sum_{i=1}^{n} A_{n}\right)$. 
Table 1 Integrated Models for Prisms.

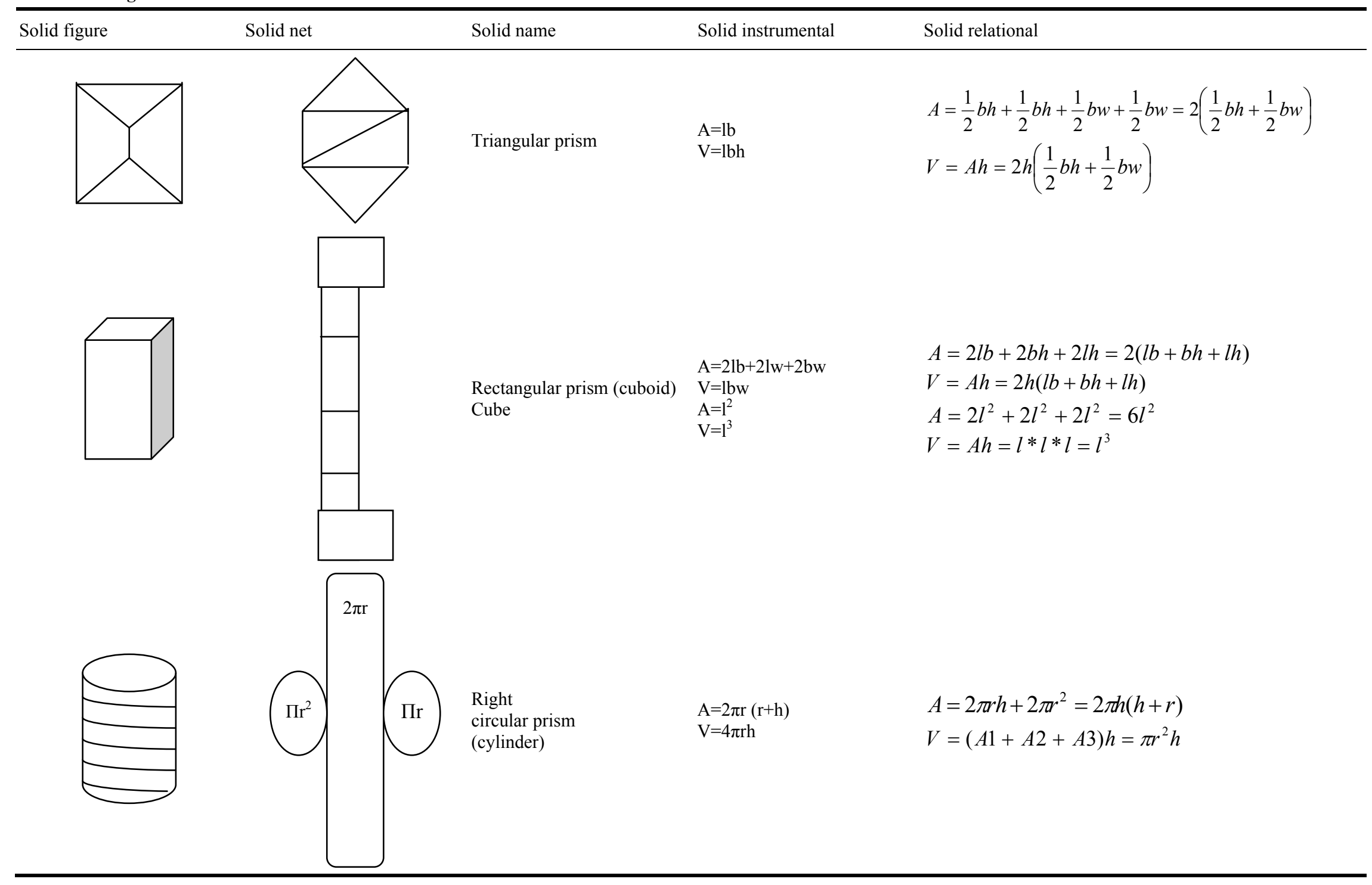


Table 2 Integrated Models for Pyramids.

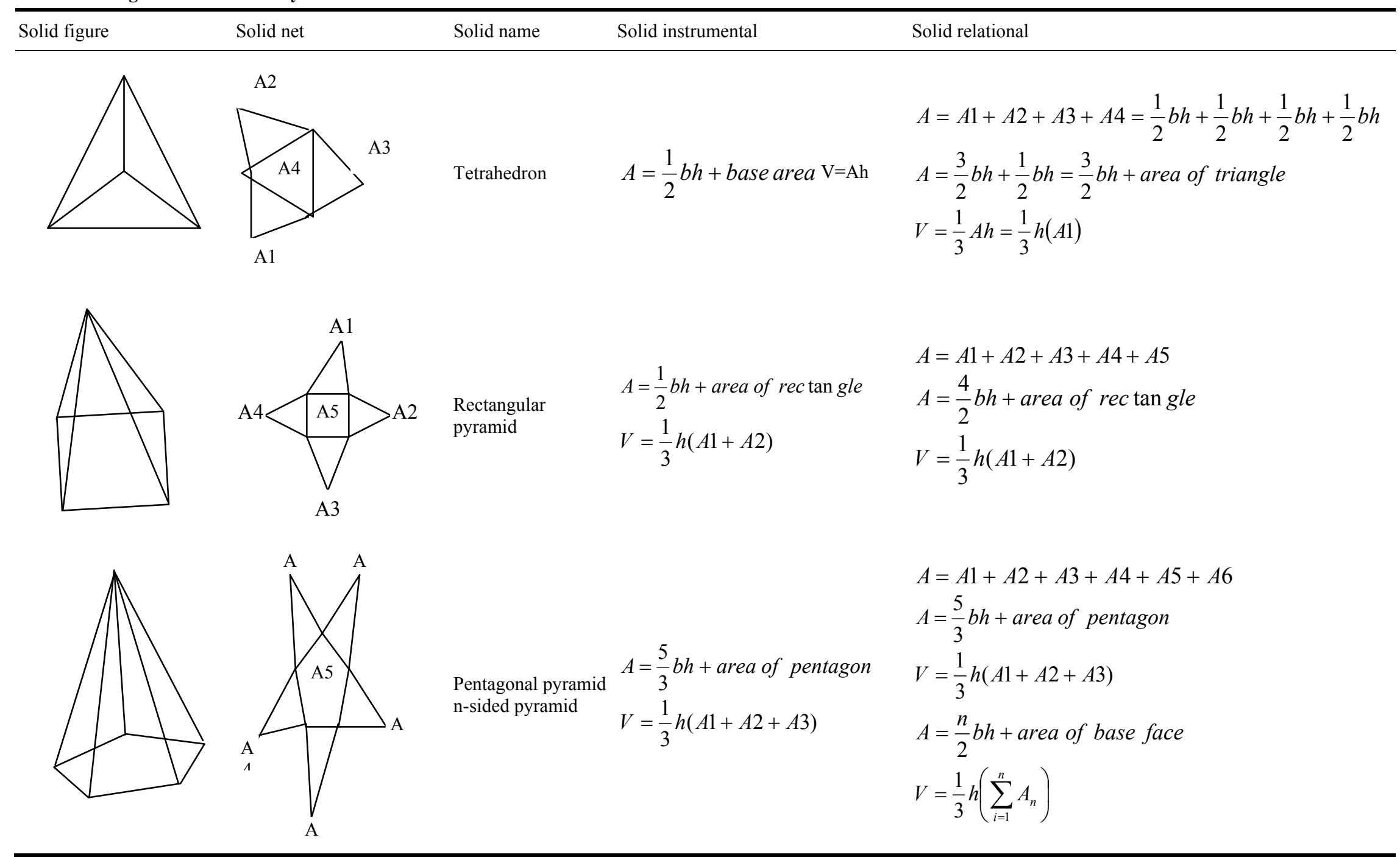

Source: TIMES (2011) 
Table 3 Integrated Models for Sphere.

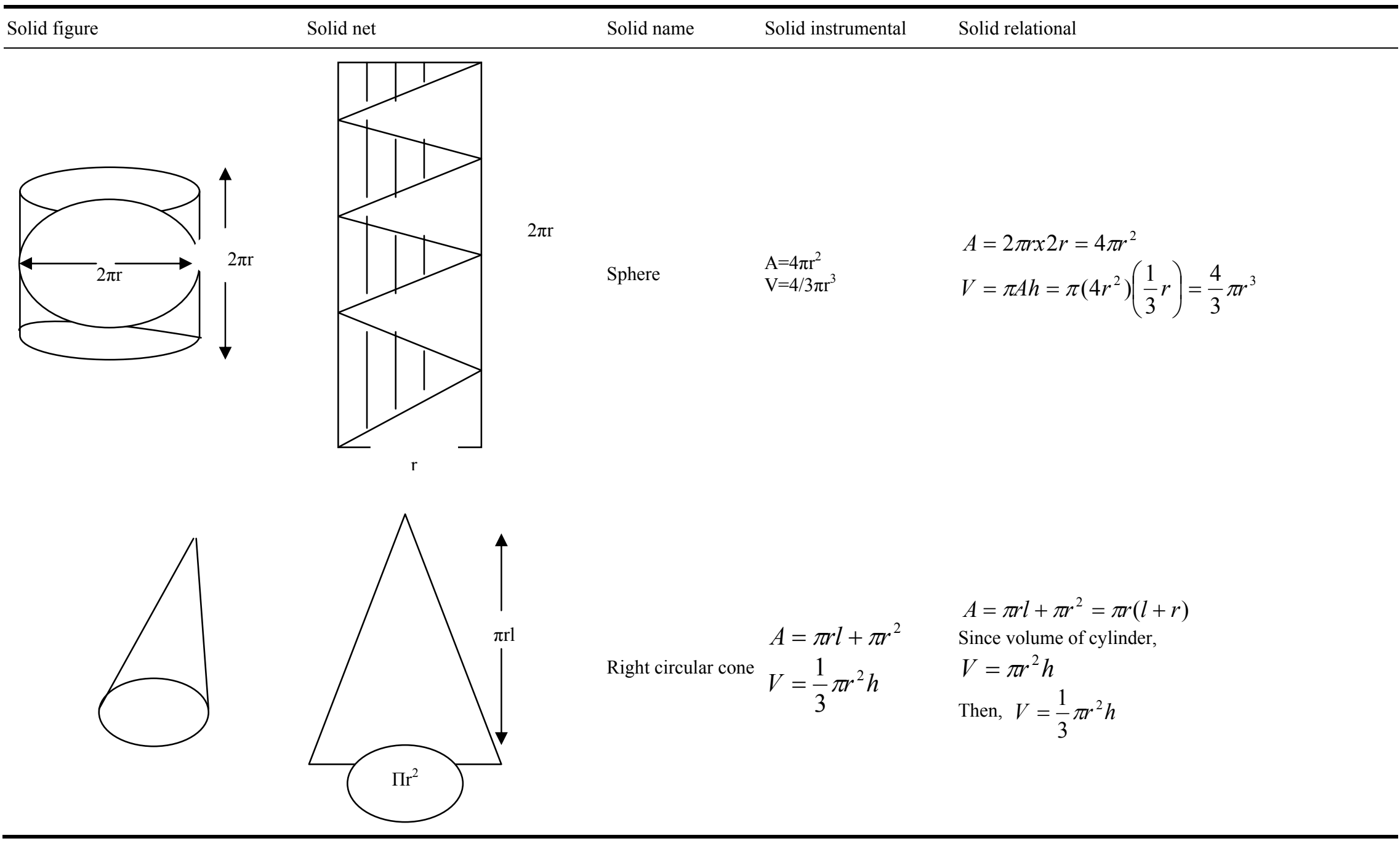

Source: TIMES (2011) 
Table 4 Scores of Integrated Design Model.

\begin{tabular}{lll}
\hline & Relational & Instrumental \\
\hline Mean & 46.59 & 47.50 \\
Variance & 89.02 & 121.43 \\
Observations & 122 & 122 \\
Pearson Correlation & 0.525 & 0.86 \\
Hypothesized Mean Difference & 0 & 0 \\
df & 121 & 121 \\
t Stat & 10.36 & 9.73 \\
$\mathrm{P}(\mathrm{T}<=\mathrm{t})$ one-tail & 0.000 & 0.000 \\
$\mathrm{t}$ Critical one-tail & 1.72 & 1.89 \\
\hline
\end{tabular}

The results of table 3 show the order in which preservice teachers grouped the spheres in the integrated design models. They applied their instrumental knowledge in circles and cylinders to solve for the spheres and cones. Instrumentally, the total surface area of the sphere with radius $\mathrm{r}$ is $\mathrm{A}=4 \pi r^{2}$, the hemisphere (which is half of the sphere) to be $2 \pi r^{2}$, and the cone was $\mathrm{A}=\pi r(1+r)$. The volume of the sphere was $\mathrm{V}=4 / 3 \pi r^{3}$, hemisphere $\mathrm{V}=2 / 3 \pi r^{3}$, and cone $\mathrm{V}=1 / 3 \pi r^{2} h$.

In deriving the relational models, the total surface area of a sphere of radius $r$ was understood to be 4 times the area of a circle of radius $r$ or $4 \times\left(\pi r^{2}\right)$, and that of the cone is the sum of lateral side and circular or $\pi \mathrm{rl}+\pi \mathrm{r}^{2}$. The volume of the sphere is obtained by filling the container up to the brim with water, carefully place one of the spheres in the container for some of the water to over flow into the trough, pour out the water from the trough into a measuring cylinder and measure the water over flowed. It would be noted that the volume is nearly equal to the measure of the volume of the water displaced (over flowed) by the sphere, and which was $\mathrm{V}=\pi 4 / 3 \mathrm{r}^{3}$.

The curved surface area of a hemisphere is half the surface area of the sphere, which is $2 \pi r^{2}$. That means the two faces of the hemisphere, which is the total surface area, is $\mathrm{A}=2 \pi r^{2}+\pi r^{2}$. To find the volume, we took spheres, big container, and large trough to place the container. Since the volume of a hemisphere is half that of a sphere, then we appropriately arrived at the volume of the hemisphere $\mathrm{V}=2 / 3 \pi r^{3}$.
To find the volume of the cone, we filled the cone up to the brim with sand, and emptied it into the cylinder. When the cone was filled, and emptied into the cylinder for the third time, the cylinder became full. This justifies that three times the volume of a cone, makes the volume of a cylinder, or $\mathrm{V}=1 / 3 \pi r^{2} h$.

The paired-samples t-test was conducted to compare the mean scores for the instrumental and relational learning models. There was no statistically significant difference [p_value $=0.000<\alpha=0.05$ ] between the models. It is still worthy of critique that the preservice teachers' scores in the relational models could have been affected by history, interest in activities, self-motivation in the involvement and properly laid down instructional algorithms, and was not entirely adjudged to be more effective than the instrumental models. This argument stems from our observation that the instrumental tasks were completed far earlier than scheduled, and that could mean the preservice teachers understood those models too. It was therefore, difficult to superimpose the superiority of particular learning models on the preservice teachers. The two models are also intrinsically linked and mutually dependent on each other. For instance, in deriving the nets, preservice teachers conjectured and memorized formulas from two-dimensional shapes and figures, and this is clearly instrumental. In performing higher manipulations, the preservice teachers needed practical and systematical instructions to arrive at the models, because most of them write down formulas before deriving the total surface areas and volumes. 


\section{Conclusions}

The preservice teachers successfully and effectively engaged in both instrumental and relational tasks to solve problems involving total surface areas and volumes of solids. It is therefore, prudent to integrate the formulas and fixed procedures with systematical and sequential conceptualizing of the prelations. We recommended that:

Classroom instructional discourse should neither be aligned to instrumental nor relational; rather stakeholders should integrate the two styles to espouse all views.

Relational views is sacrosanct with stocking the classroom like a carpenter's shop, and strictly calling for instrumental learning means that the mathematics classroom should mimic the tenets of a court room, where everybody must provide quotations to back the solutions of problems. There must be therefore, be an integrated design model to satisfy these dichotomous classes of learning.

Teacher training institutions should encourage instructors to devise and improvise integrated instructional designs in the processes. By recalling and memorizing a formula help in discovering how the formula came into being, and the vice versa.

The curriculum review and development authorities or boards incorporate integrated design models in the mathematics classroom, and sensor those models. This would benefit examination candidates who need little relational learning, and equally benefit technical candidates who are more oriented in practical relational activities.

\section{References}

[1] Afram Publications Editorial Board (1981). New Mathematics for Secondary. Accra: Afram Publications Ltd.

[2] Appau, K. (2006). Mathematics for Diploma Colleges. Kumasi: RockPrint Press. Approach.

[3] Asafo-Adjei, R. (2002). Teaching Basic Mathematics for Colleges of Education. Kumasi: BAYOBA Ltd.

[4] Asiedu, P. (1997). Mathematics for Senior High Schools in West Africa. Accra: Afram Publications Ltd.
[5] Beswick, K. (2005). Preservice teachers' understandings of relational and instrumental understanding. In Chick, H. L. \& Vincent, J. L. (Eds.). Proceedings of the 29th Conference of the International Group for the Psychology of Mathematics Education, Vol. 2, pp. 161-168. Melbourne: PME.

[6] Bishop, G. (1980). Curriculum Development, A Textbook for Students. Cambridge: Cambridge University Press.

[7] Bolt, B. (1987). Even More Mathematical Activities, Cambridge: Cambridge: Cambridge University Press.

[8] Creswell, J.W. (2014). Research Design: Quantitative, Qualitative and Mixed Methods Approaches. London: Sage Publications.

[9] Crotty, M. (1998). The foundations of social research: Meaning and perspective in the research process. Thousand Oaks, CA: Sage.

[10] Egodawatte, G. (2011). Secondary School Students' Misconceptions in Algebra. A thesis submitted in conformity with the requirements for the degree of Doctor of Philosophy Department of Curriculum, Teaching and Learning Ontario Institute for Studies in Education, University of Toronto.

[11] Ernest, P. (1991). The Philosophy of Mathematics Education, London: Falmer Press. Hall Inc.

[12] Martin, J.L. (1994). Mathematics for Teacher Training in Ghana, Tutor's Notes. Accra: Unimax Publishers Ltd.

[13] Nabie. M.J. (2001). Psychology of Teaching Mathematics. Accra: Akonta Publications

[14] Ontario Ministry of Education (2008). Geometry and Spatial Sense, Grades 4 to 6: A Guide to Effective Instruction in Mathematics, Kindergarten to Grade 6. Ontario: Ministry of Education.

[15] Pesek, D.D. \& Kirshner, D. (2000). Interference of Instrumental Instruction in Subsequent Relational Learning. Journal for Research in Mathematics Education, Vol. 31, No. 5, pp.524-540. New York: National Council of Teachers of Mathematics.

[16] Rachlin, L.S., Matsumoto, N.A. \& Wada, T.A.L. (1992). Algebra I, Process. Approach. Sidney: Prentice-Hall Inc.

[17] SAIIDE (2008). Developing Understanding in Mathematics From the module: Teaching and Learning Mathematics in Diverse Classrooms. Braamfontein: Creative Commons.

[18] Skemp, R.R. (1976). Relational Understanding and Instrumental Understanding. Mathematics Teaching, 77, 20-26.

[19] Skemp, R.R. (1978). Relational understanding and instrumental understanding. Arithmetic Teacher, 26(3), 9-15.

[20] Summit, R. \& Rickards, T. (2013). A constructivist approach to mathematics laboratory classes. Lighthouse 
Delta 2013: The 9th Delta Conference on teaching and learning of undergraduate mathematics and statistics, 24-29 November 2013, Kiama, Australia.

[21] The Improving Mathematics Education in Schools (TIMES--2011). Cones, Pyramids and Spheres
(Measurement and Geometry: Module 12). University of Melbourne: Australian Mathematical Sciences Institute (AMSI). University Press.

[22] Williams, E. \& Shuard, H. (1982). Primary Mathematics Today. Sidney: Prentice-Hall. 